\title{
Cardiac sarcoidosis: Advantages and limitations of advanced cardiac imaging
}

\author{
Sabahat Bokhari, $M D,{ }^{a}$ and Tarick Sheikh, $M D^{a}$ \\ a Divison of Cardiology, Lehigh Valley Health Network, Allentown, PA
}

Received Jul 6, 2021; accepted Jul 7, 2021

doi:10.1007/s12350-021-02757-w

See related article, pp. 2132-2144

\section{WHAT IS CARDIAC SARCOIDOSIS AND HOW IS IT DIAGNOSED?}

Cardiac sarcoidosis (CS) is a localized manifestation of multisystemic granulomatous disorder of unknown etiology. It has heterogeneous manifestations depending on which organs are involved. Although the overall prognosis is good, cardiac involvement generally portends a poor prognosis and has been found to contribute to significant morbidity and mortality. Although the prevalence of clinical cardiac involvement is estimated near $5 \%$, difficulties faced with accurate diagnosis contribute to an overall underestimation of disease burden. This is supported by prior autopsy data that suggest the prevalence of cardiac involvement nearing 20-25\% when accounting for sub-clinical cases. $^{1,2}$

The current diagnostic criteria are based on the modified Japanese Ministry of Health and Welfare (JMHW) guidelines published in 2006 and the Heart Rhythm Society (HRS) consensus statement published in 2014. Both guidelines involve a histological diagnosis by endomyocardial biopsy or objective recognition of specific clinical or imaging features. ${ }^{3,4}$ Each of these modalities have been found to carry limitations, and therefore the focus has shifted toward utilizing advanced imaging modalities such as cardiac magnetic resonance (CMR) and 18F-fluorodeoxyglycose (FDG) positron emission tomography (PET) as important tools to improve the diagnostic certainty and optimize management of CS.

\footnotetext{
Reprint requests: Sabahat Bokhari, MD, Divison of Cardiology, Lehigh

Valley Health Network, Allentown, PA ; cardiomd2020@gmail.com J Nucl Cardiol 2022;29:2145-8.

$1071-3581 / \$ 34.00$

Copyright (C) 2021 American Society of Nuclear Cardiology.
}

A major limitation in the evaluation of CS is that no gold standard clinical diagnostic criteria exist. Although a positive endomyocardial biopsy is definitive for diagnosing CS, the sensitivity of this technique is limited (30\%) due to patchy cardiac involvement.

\section{HOW CAN ADVANCED CARDIAC IMAGING MODALITIES BE UTILIZED IN THE DIAGNOSIS AND MANAGEMENT OF CARDIAC SARCOIDOSIS?}

CMR relies on identifying the presence of patchy or focal late gadolinium enhancement (LGE) in the subepicardial and mid-myocardial wall. No specific pattern of LGE on CMR is diagnostic for CS. Importantly, CMR and FDG-PET evaluate different pathologic processes, namely fibrosis via LGE in the former and inflammation via labeled glucose uptake of activated macrophage in the latter, and neither technique is specific for fibrosis or inflammation caused by cardiac sarcoidosis. As such, these different advanced imaging tests may provide complementary information for the appropriately selected patient in whom clinical suspicion for cardiac sarcoidosis warrants further evaluation.

Advantages of FDG-PET relative to CMR include the following:

1. It is preferred in patients with claustrophobia, cardiac pacemakers, and implantable cardioverter-defibrillator devices and in patients with advanced kidney disease.

2. Diagnosis of early-stage cardiac sarcoidosis may be facilitated by FDG-PET which may precede detectable LGE by CMR.

3. The prognostic value of abnormal FDG appears to be strongest in the presence of a concurrent resting myocardial perfusion, which would correspond to the presence of scar.

4. The FDG PET is useful for monitoring of ongoing inflammation in response to therapy. 
Limitations of FDG-PET relative to CMR include the following:

1. The non-inflammatory myocardial FDG uptake even with optimal preparations of FDG-PET scans.

2. Atrial fibrillation and bundle branch block could affect the regional glucose utilization.

3. Exposure to radiation.

Although there are no large randomized controlled prospective studies evaluating the efficacy of high-dose glucocorticoids in CS, retrospective studies suggest that steroid therapy is associated with improved outcomes related to specific endpoints including LVEF and death. ${ }^{5}$ Current follow-up of patients on corticosteroid therapy typically includes annual Holter monitoring and transthoracic echocardiography to evaluate for changes in ejection fraction or arrhythmia burden. ${ }^{6}$ However, these methods are less sensitive for detecting subtle clinical changes as compared to more advanced imaging modalities such as FDG-PET imaging or CMR.

Osborne et al. performed a prospective evaluation of 23 patients with cardiac sarcoidosis followed over a median of 2 years, the majority of whom were treated with corticosteroids (91\%), angiotensin-converting enzyme/angiotensin receptor blocker (78\%), and betablockers (83\%). All patients had at least two PET exams at different time points, during which FDG uptake was measured using maximum standardized uptake value and standardized uptake value volume. The authors identified a significant inverse relationship between LVEF and standardized uptake value measurements. Additionally, non-responders to therapy (identified by changes in FDG uptake) had a significant decrease in LVEF compared with responders who demonstrated an increase in LVEF.?

The initial decision to pursue advanced imaging evaluation for cardiac sarcoidosis is based upon the presence of following specific concerning features:

1. Presence of idiopathic high-degree AV block in patients less than age 55

2. Monomorphic ventricular tachycardia of unknown etiology.

3. Prior diagnosis of extracardiac sarcoidosis with raised clinical suspicion for cardiac involvement.

We propose the following advanced algorithm for the diagnosis and management of cardiac sarcoidosis as shown in Figure 1.

\section{PATIENT PREPARATION FOR FDG-PET IMAGING AND CATEGORIZATION OF IMAGING FINDINGS}

Initially, the patient undergoes resting myocardial perfusion imaging with PET (Ammonia N-13 or Rubidium-82) or SPECT (Tc-99m Sestamibi or Tc-99m Myoview) followed by F-18 FDG imaging.

The preparation for FDG-PET imaging requires that the patient's fasting plasma glucose is less than $200 \mathrm{mg} /$ $\mathrm{dl}$ and the patient has remained on a high-fat/no or lowcarbohydrate diet for a period of 18-24 hours. Additionally, 10 to $50 \mathrm{IU} / \mathrm{kg}$ of intravenous unfractionated heparin is administered 15 minutes prior to FDG injection. Scans are then obtained after a 90-120-minute incubation time. ${ }^{8}$ Areas of pathologic glucose uptake (inflammation) are then identified on PET imaging and compared with myocardial perfusion imaging. ${ }^{3}$ The diagnostic accuracy for FDG-PET is dependent on the appropriate suppression of physiologic glucose utilization by normal myocardium, as facilitated by patient compliance with a high-fat/no or low-carbohydrate diet and intravenous unfractionated heparin.

The categorization of the imaging findings is divided into four separate categories (Figure 2).

1. Normal: normal perfusion and normal FDG uptake.

2. Early disease: no or mild perfusion defect with increased corresponding FDG uptake.

3. Progressive disease: moderate perfusion defect with increased corresponding FDG uptake.

4. Fibrous disease: severe perfusion defect with minimal or no FDG uptake.

\section{WHAT DID WE LEARN FROM THE CURRENT PUBLICATION?}

Bekki et al showed that the localization of myocardial FDG uptake on positron emission tomography (PET) and myocardial perfusion abnormality in corticosteroid-naïve CS provides long-term prognostic risk stratification.

Bekki et al have addressed the utility of FDG-PET in the diagnosis and management of CS. They identified a corticosteroid-naïve patient population with clinical suspicion of cardiac sarcoidosis who underwent FDGPET imaging for assessment of cardiac sarcoidosis. All patients followed a high-fat/low-carbohydrate diet with at least 18 hours of fasting prior to PET scanning, with a single IV bolus injection of FDG (4.2 MBq $(0.12 \mathrm{mCi}) /$ $\mathrm{kg}$ body weight). After a one-hour incubation time, PET imaging was performed, and additionally patients also underwent Tc-99m Sestamibi SPECT scintigraphy. Patients with focal or focal on diffuse FDG uptake pattern were studied. Standardized uptake values 


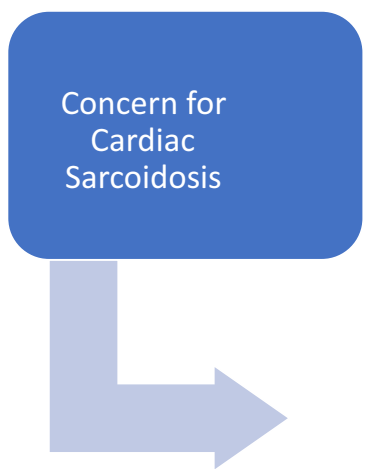

- Concerning features include:

- - Age <55 with idiopathic high degree AV-block.

- - Idiopathic monomorphic ventricular tachycardia.

- Known diagnosis of extra-cardiac sarcoidosis with

clinical, ECG or echo clues for cardiac involvement.

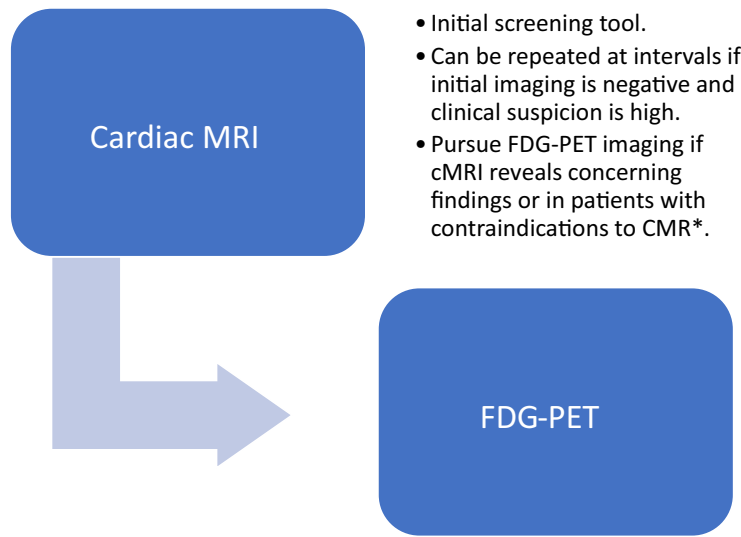

- Useful for diagnosis and monitoring response to therapy.

- Repeated after 8-12 weeks to assess response to therapy.

Figure 1. Proposed clinical algorithm for the use of advanced cardiac imaging for the diagnosis and monitoring of cardiac sarcoidosis. *Contraindication to CMR (i.e., claustrophobia, cardiac device, or advanced renal dysfunction).

\begin{tabular}{|c|c|c|c|}
\hline Disease Category & Uptake Pattern & Perfusion & Metabolism \\
\hline Normal & $\begin{array}{l}\text { Perfusion: Normal } \\
\text { Metabolism: No FDG } \\
\text { Uptake }\end{array}$ & & \\
\hline Mild or Early Disease & $\begin{array}{c}\text { "Focal Mismatch Pattern" } \\
\text { Perfusion: No or mild } \\
\text { defect } \\
\text { Metabolism: FDG uptake } \\
\text { in area of defect }\end{array}$ & & \\
\hline $\begin{array}{c}\text { Moderate or } \\
\text { Progressive Disease }\end{array}$ & $\begin{array}{l}\text { "Eocal Mismatch Pattern" } \\
\text { Perfusion: Moderate } \\
\text { defect } \\
\text { Metabolism: FDG uptake } \\
\text { in area of defect }\end{array}$ & & \\
\hline $\begin{array}{l}\text { Severe or Fibrous } \\
\text { Disease }\end{array}$ & $\begin{array}{l}\text { Perfusion: Severe defect } \\
\text { Metabolism: No or } \\
\text { minimal FDG uptake }\end{array}$ & & \\
\hline
\end{tabular}

Figure 2. Classification of cardiac PET perfusion/metabolism image patterns and correlation with disease stage in cardiac sarcoidosis. 
(SUVs) and segmental FDG intensity index were calculated for each patient. In addition, right ventricular FDG uptake was also assessed. The authors found that right ventricular (RV) FDG uptake was more frequent in patients who suffered adverse event than those without $\mathrm{RV}$ uptake, during a mean follow-up period of 4.9 years. The survival rate free from AEs was significantly lower in patients with FDG uptake in the basal anterolateral LV wall than those without it.

Right ventricular involvement has been described in sarcoidosis since the late 1970's on thallium scintigraphy $^{9}$ and more recently on cardiac MRI and FDG PET. ${ }^{10}$ Right ventricular abnormalities (RV systolic dysfunction and late gadolinium enhancement) on cardiovascular magnetic resonance imaging in patients with sarcoidosis have been shown to have adverse outcomes in patients with sarcoidosis. ${ }^{11}$

Despite these results, the study had several limitations. First, this is a single-center observational study with a small sample size. Although we do learn that regional variations in FDG uptake on PET imaging continues to provide important prognostic value in patients with corticosteroid-naïve cardiac sarcoidosis, the relatively small sample size studied by the authors raises a question of how easily these results could be generalized to various patient populations. Additionally, PET imaging in this study was obtained after 60 minutes of FDG injection rather than the more commonly used 90-120 minutes delay and patients did not receive IV heparin prior to FDG injection, ${ }^{8}$ this may have affected the suppression of non-inflammatory myocardial FDG uptake.

From this article, we can gather that RV and/or basal anterolateral LV segmental uptake with concomitant perfusion abnormalities are useful prognostication parameters for risk assessment and stratification in patients with corticosteroid-naïve CS. This further supports the expert consensus that FDG-PET imaging in CS is a vital tool in the early identification of clinical disease, ${ }^{6}$ allowing for improved outcomes with early initiation of therapy with corticosteroids and even early implantation of ICDs for primary prevention. FDG-PET is also useful in evaluation of response to treatment in established CS patients. CMR and FDG-PET provide complementary information in suspected CS patients. Additionally, the diagnostic tests must be individualized to the specific patient and local expertise of the center performing the tests.

\section{References}

1. Blankstein R, Waller AH. Evaluation of known or suspected cardiac sarcoidosis. Circ Cardiovasc Imaging 2016;9:e000867.

2. Skali H, Schulman AR, Dorbala S.18F-FDG PET/CT for the assessment of myocardial sarcoidosis. Curr Cardiol Rep 2013;15:352

3. Hiraga H, Yuwai K, Hiroe M. Diagnostic standard and guidelines for sarcoidosis. Jpn J Sarcoidosis Granulomatous Disord 2007;27:89-102

4. Birnie DH, Sauer WH, Bogun F et al. HRS expert consensus statement on the diagnosis and management of arrhythmias associated with cardiac sarcoidosis. Heart Rhythm 2014;11:130523

5. Sadek MM, Yung D, Birnie DH, Beanlands RS, Nery PB. Corticosteroid therapy for cardiac sarcoidosis: a systematic review. Can J Cardiol 2013;29:1034-41

6. Hulten E, Aslam S, Osborne M, Abbasi S, Bittencourt MS, Blankstein R. Cardiac sarcoidosis-state of the art review. Cardiovasc Diagn Ther 2016;6:50-63

7. Osborne MT, Hulten EA, Singh A, Waller AH, Bittencourt MS, Stewart GC, et al. Reduction in 18F-fluorodeoxyglucose uptake on serial cardiac positron emission tomography is associated with improved left ventricular ejection fraction in patients with cardiac sarcoidosis. J Nucl Cardiol 2014;21:166-74

8. Morooka M, Moroi M, Uno K, Ito K, Wu J, Nakagawa T, et al. Long fasting is effective in inhibiting physiological myocardial 18F-FDG uptake and for evaluating active lesions of cardiac sarcoidosis. EJNMMI Res 2014;4:1

9. Buckley B, Rouleau J, Whitaker J, Strauss H, Pitt B. The use of 201 Thallium for myocardial perfusion imaging in sarcoid. Chest 1977;72:27-32

10. Blankstein R, Osborne M, Naya M, Waller A., Kim CK, Murthy VL, et al. Cardiac positron emission tomography enhances prognostic assessments of patients with suspected cardiac sarcoidosis. J Am Coll Cardiol 2014;63:329-36

11. Velangi P, Chen K, Kazmirczak F, Okasha O, Wald L, Roukoz H, et al. Right ventricular abnormalities on cardiovascular magnetic in patients with sarcoidosis. JACC Cardiovasc Imaging 2020;13:1395-405

Publisher's Note Springer Nature remains neutral with regard to jurisdictional claims in published maps and institutional affiliations. 